\title{
El legado escrito de Florence Nightingale. Un rico patrimonio de la profesión enfermera
}

\section{The Written Legacy of Florence Nightingale. A rich heritage of the nursing profession \\ O legado escrito de Florence Nightingale. Uma rica herança da profissão de enfermagem}

\section{Antonio Claret García Martínez ${ }^{1}$}

\section{${ }^{1}$ Doctor en Historia. Profesor Titular de Universidad. Facultad de Humanidades. Universidad de Huelva.} Correspondencia: Facultad de Humanidades. Avda. Tres de Marzo s/n 21071 (Huelva).

Correo electrónico de contacto: antonio.garcia@dhis2.uhu.es

Cómo citar este artículo: García Martínez, A.C. (2021). El legado escrito de Florence Nightingale. Un rico patrimonio de la profesión enfermera. Cultura de los Cuidados (Edición digital), 25 ( $\mathrm{N}^{\circ}$ esp.). Recuperado de http://dx.doi.org/10.14198/cuid.2021.esp.07

\section{RESUMEN}

El presente estudio ofrece un acercamiento al entorno de cultura escrita en el que se desenvolvió la enfermera británica Florence Nightingale durante toda su vida, que mostró una pasión creciente por la escritura. Se comunicó a través de cartas con personas de las más altas esferas de la sociedad victoriana (políticos, intelectuales y científicos), además de mantener una estrecha relación con su familia. Compuso y publicó numerosas obras para el ámbito sanitario y utilizó sistemáticamente el poder omnímodo de la prensa para difundir su obra. Contó con la ayuda inestimable de amigos poderosos e influyentes y en la actualidad su legado escrito se encuentra muy disperso por instituciones de distintos países, siendo en la actualidad una parte de él digitalizado y 
Revista científica de la Asociación de Historia y Antropología de los Cuidados (Universidad de Alicante)

disponible en internet, con lo que su difusión y estudio permite un mejor conocimiento de la personalidad de la ilustre enfermera británica. Su figura traspasó los límites de la práctica enfermera y circuló por buena parte de Europa la imagen de mujer luchadora e incansable por los derechos de la mujer, apareciendo con frecuencia en la prensa decimonónica de España con rasgos de heroína internacional y modelo a seguir.

La escritura en todas sus manifestaciones había jugado un papel determinante en la configuración de la imagen de Florence Nightingale.

Palabras clave: Florence Nightingale; enfermera; cultura escrita; fuentes escritas.

\section{ABSTRACT}

This study offers an approach to the environment of written culture in which Florence Nightingale developed throughout her life, showing a true passion for writing. She communicated through letters with people from the highest social strata of Victorian society, politicians, intellectuals and scientists, in addition to maintaining a close relationship with her family. She composed and published many works for the health field and systematically used the allencompassing power of the press to spread her work. He had the invaluable help of powerful and influential friends and today his written legacy is widely dispersed by institutions in different countries and is being digitized and hosted on the internet, so its dissemination and study allows a better understanding of the personality of the illustrious British nurse.

Her figure went beyond the limits of nursing practice and the image of a woman who fought and tireless for women's rights circulated throughout Europe, frequently appearing in the nineteenth-century press in Spain with traits of an international heroine and role model.

Writing in all its manifestations had played a decisive role in shaping Florence Nightingale's image.

Keywords: Florence Nightingale; nurse; written culture; written sources.

\section{RESUMO}

O presente estudo oferece uma abordagem ao ambiente da cultura escrita em que Florence Nightingale se desenvolveu ao longo de sua vida, demonstrando uma verdadeira paixão pela escrita. Ele se comunicou por meio de cartas com pessoas dos mais altos escalões da sociedade vitoriana, políticos, intelectuais e cientistas, além de manter um relacionamento próximo com sua família. Ele compôs e publicou muitos trabalhos para a área da saúde e usou sistematicamente o poder abrangente da imprensa para divulgar seu trabalho. Contou com a ajuda inestimável de amigos poderosos e influentes e hoje seu legado escrito está amplamente disperso por instituições de diversos países e está sendo digitalizado e hospedado na internet, com a qual sua divulgação e estudo permitem um melhor conhecimento da personalidade de a ilustre enfermeira britânica. Sua figura ultrapassou os limites da prática da enfermagem e a imagem de uma mulher lutadora e incansável pelos direitos da mulher circulou por toda a Europa, aparecendo com frequência na imprensa do século XIX na Espanha com traços de heroína internacional e modelo.

A escrita em todas as suas manifestações desempenhou um papel determinante na formação da imagem de Florence Nightingale.

Palavras-chave: Florence Nightingale; enfermeira; cultura escrita; fontes escritas.

\section{Florence Nightingale y su pasión por la lectura y la escritura}

Florence Nightingale manifestó una 
apasionada atracción por la escritura desde su infancia, pasión que no haría más que crecer con el paso de los años. De una fecha tan temprana como 1829 se conserva una carta de Florence Nightingale escrita con tan sólo nueve años. Cuando la ilustre enfermera británica falleció el día 20 de agosto de 1910, el número de sus cartas y escritos se contaba por millares e instituciones de todo el mundo se enorgullecen de tener entre sus fondos documentales textos de Mss. Nightingale.

Imagen 1. Portada de la obra "The Life of Florence Nightingale”, primera biografía de la enfermera británica.

Edward Tyas Cook recoge en su obra La vida de Florence Nightingale (1913) que "Miss Nightingale era una mujer acumuladora (de papeles), y hasta que llegó a los 90 años de edad, la acumulación de papeles almacenados en su casa en el momento de su fallecimiento era muy grande” (Imagen 1).

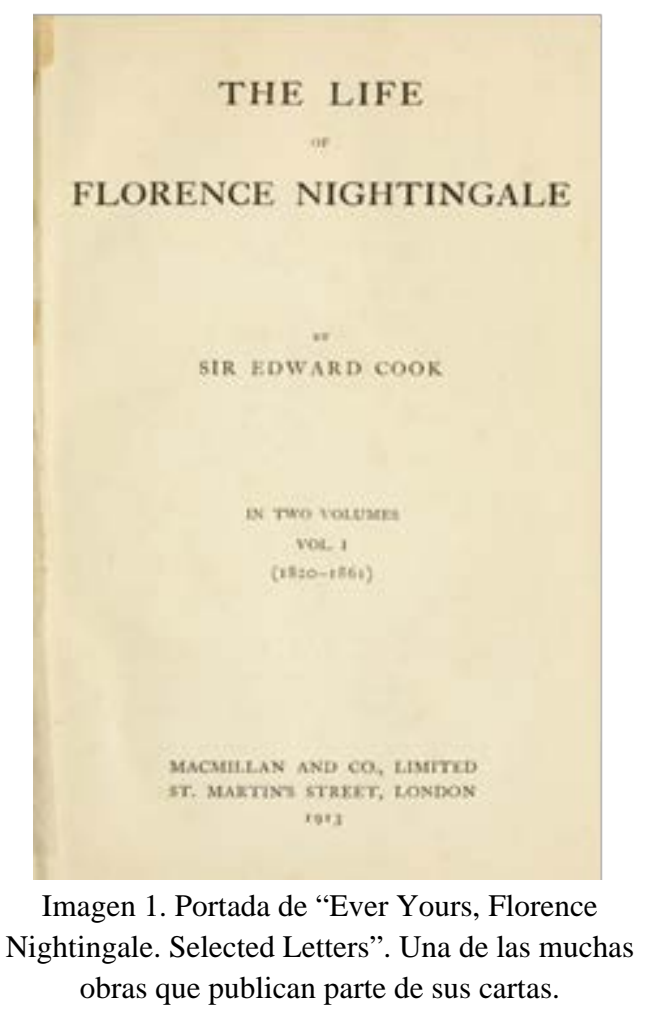

Y esta pasión la cultivó hasta los últimos días de su vida. Además de miles de cartas, también escribió alrededor de 200 trabajos, entre libros, informes sobre hospitales $\mathrm{y}$ folletos; sobre saneamiento y otros temas relacionados con la salud, además de contribuir al campo de las estadísticas.

A lo largo de su vida, brindó asesoramiento sobre una variedad de problemas de atención médica a especialistas de todo el mundo (Backes, V. M., 2000).

Aunque enferma y postrada en cama durante una parte de su vida, Florence Nightingale continuó su gran trabajo a través del empleo constante de la correspondencia. Sus cartas conservadas se cuentan por millares. Con el aumento de prestigio de la enfermera a todo lo largo del siglo XX y el desarrollo de las nuevas tecnologías digitales, desde comienzos del siglo XXI, las más diversas instituciones han iniciado proyectos de conservación y difusión del patrimonio escrito producido por Florence Nightingale, poniendo a disposición de los investigadores y de un público más general un valioso acervo documental que está permitiendo abordar planos muy diferentes de la personalidad de la ilustre británica, que van mucho más alla de su faceta más visible: la de enfermera. Mujer culta, apasionada del arte y de las ciencias, como las matemáticas; inquieta por temas sociales, e incómoda por las ataduras que le imponían a la mujer los convencionalismos de la etapa victoriana, en sus cartas y escritos se despliega toda su poderosa personalidad (Cook, E., 1913).

Cuidó mucho sus relaciones sociales y mantuvo una correspondencia asidua con personajes destacados de la alta política, la economía, la cultura y la sociedad británica de su época, manifestando un uso magistral del intercambio de correspondencia como instrumento no sólo de comunicación entre partes, sino también para mover voluntades y conseguir objetivos para su causa (Baly, M. E., 1988) (Imagen 2). 

(Universidad de Alicante)

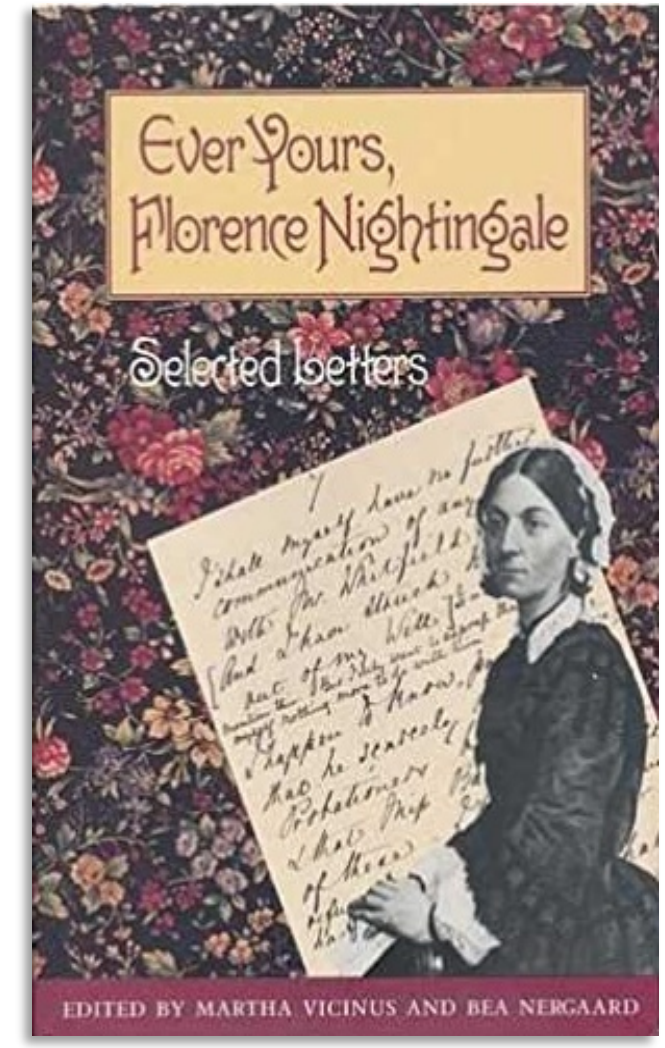

Imagen 2. Portada de "Ever Yours, Florence Nightingale. Selected Letters”. Una de las muchas obras que publican parte de sus cartas.

Cronología de las principales obras escritas por florence nightingale

-1828. Autobiografías escritas con 8 años para trabajos de clase.

-1842 y siguientes. Cassandra.

-1858. Notas sobre los Hechos que afectan a la Salud, Eficiencia y Administración Hospitalaria o de la Armada Británica.

-1858. Mortalidad de la Armada Británica, en Casa y en el Extranjero, y durante la Guerra Rusa.

-1859. Notas sobre las condiciones sanitarias de los hospitales y sobre los defectos en la construcción de las salas de estos.

-1859. Notas sobre Hospitales.

-1859. Notas para Enfermería: Lo que es y Lo que no es.

-1859. Una Contribución a la Historia Sanitaria de la Armada Británica durante la última guerra con Rusia: Ilustrado con

Tablas y Diagramas.

-1860. Sugerencias de Pensamiento para los

Buscadores de la Verdad entre los Artesanos de Inglaterra.

-1862. La Administración Sanitaria de la Armada y su Reforma bajo el anterior Lord Herbert.

-1863. Observaciones sobre la Evidencia contenida en los Informes estacionales presentados a la Comisión Real sobre el Estado Sanitario de la Armada en la India. -1871. Notas Introductorias sobre las Instituciones para los Partos.

Entre otros escritos con fuerte fundamento didáctico e informativo (Nightingale, F., 1976), (Nightingale, F., 2000b).

\section{La correspondencia de Florence} Nightingale: un extraordinario legado escrito que no deja de crecer

El desarrollo de la popularidad de la enfermera británica ha tenido como una de sus consecuencias la valoración de todo su legado escrito, lo cual ha repercutido en el interés de su obra y en la puesta en marcha de proyectos de digitalización de sus textos, ya sean cartas u obras y escritos muy diversos de Enfermería (Hartill, R., 1996), (Jowett, B., 1987).

$\mathrm{Al}$ ingente número de publicaciones que, especialmente desde su muerte, comenzó a salir de imprentas de todo el mundo, se viene sumando desde finales del siglo XX un creciente número de proyectos de compilación y difusión de sus escritos a través de la digitalización de de todo su legado. Bibliotecas de universidades y distintas instituciones están difundiendo los fondos escritos de la enfermera británica a traves de proyectos de digitalización y difusión en red de este patrimonio de incalculable valor (Nightingale, F., 1914), 
(Nightingale, F., 1974) (Ilustración 3).

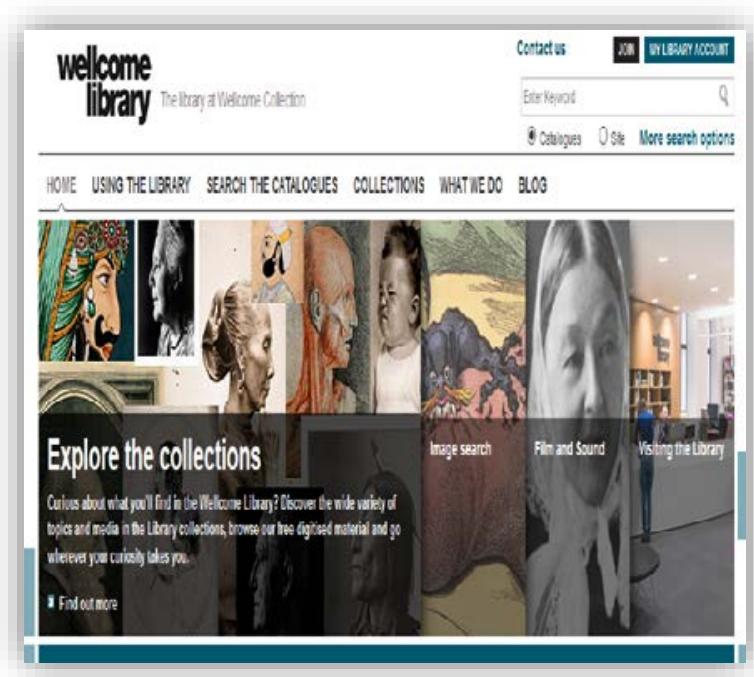

Ilustración 3. Portal de la Wellcome Library. Fragmento de la página de inicio del proyecto desarrollado por la Wellcome Library de Londres con importantes colecciones digitalizadas, entre ellas una parte de la correspondencia enviada por Florence Nightingale.

Es ahora mejor que nunca cuando se está conociendo la correspondencia mantenida durante toda su vida gracias a esos proyectos de digitalización y que, en su conjunto, ofrecen de forma nítida y ponen a disposición de las personas interesadas millares de cartas que escribió la enfermera británica. Aquí nos detendremos en aquellos proyectos que hemos utilizado para nuestro estudio.

La Welcome Library de Londres está desarrollando desde hace unos años un interesante proyecto basado en la digitalización y difusión de más de 600 cartas manuscritas que Florence Nightingale intercambió con personajes de su época. Un legado de un extraordinario valor para conocer diversos aspectos de su vida y su obra (Imagen 3).

Entre las cartas digitalizadas se encuentra una de especial valor por su singularidad. Es la carta que Florence Nightingale escribió a su primo Henry cuando tenía tan sólo 9 años.
Es una muestra más de la precoz pasión que la futura enfermera sentía por la escritura (Imagen 4).

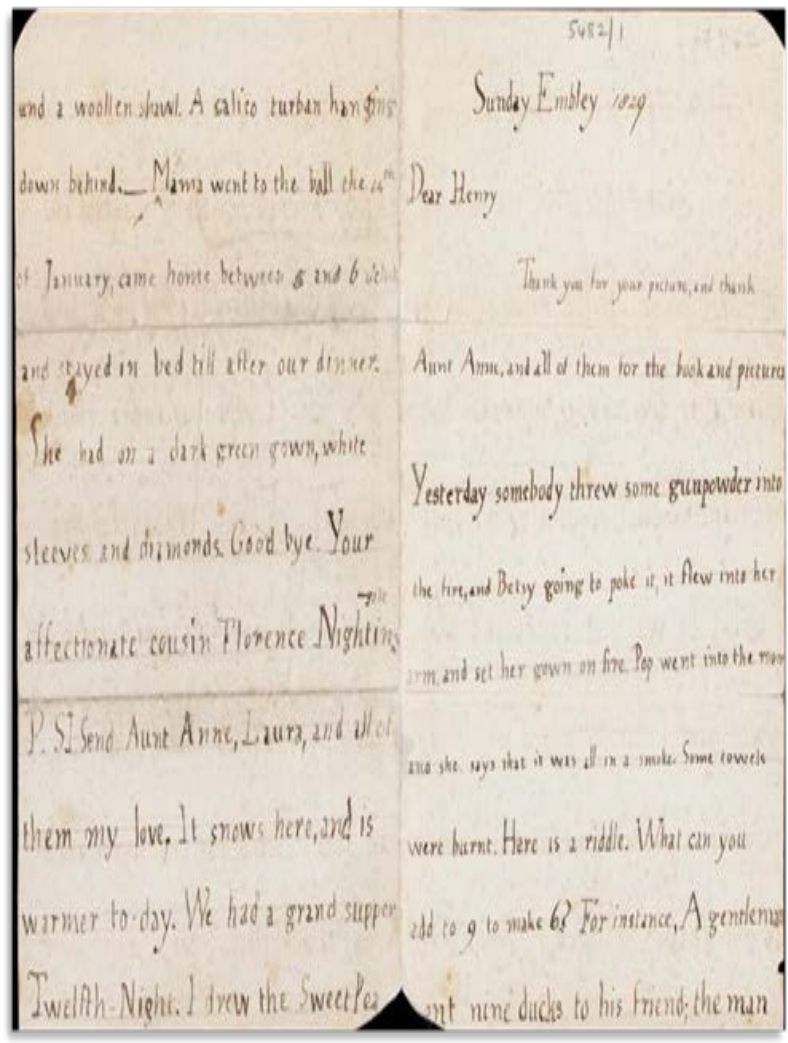

Imagen 4. Carta de Florence Nightingale a su primo Henry cuando ella contaba con 9 años (1829) (Wellcome Library Project).

La colección de la Wellcome Library comprende, principalmente, la correspondencia de Florence Nightingale ya sea en forma original o copias de las originales. La fecha abarca la totalidad de su vida y los temas tratados van desde sus intentos de convertirse en enfermera, su servicio en la guerra de Crimea y su trabajo posterior en Londres, preocupándose por la formación de las jóvenes estudiantes y toda la práctica de la Enfermería (Imagen 5). Desarrolló una ingente tarea a través de la publicación de obras referentes a la necesidad de la mejora de los hospitales y enfermerías, basándose en sus amplios conocimientos matemáticos y estadísticos, lo que influyó poderosamente en la sociedad británica y consiguió la toma de conciencia de la sociedad británica de la importancia de 
sus propuestas. Las cartas de esta colección, fechadas entre 1829 y 1910, van dirigidas a familiares, amigos y autoridades políticas, principalmente.

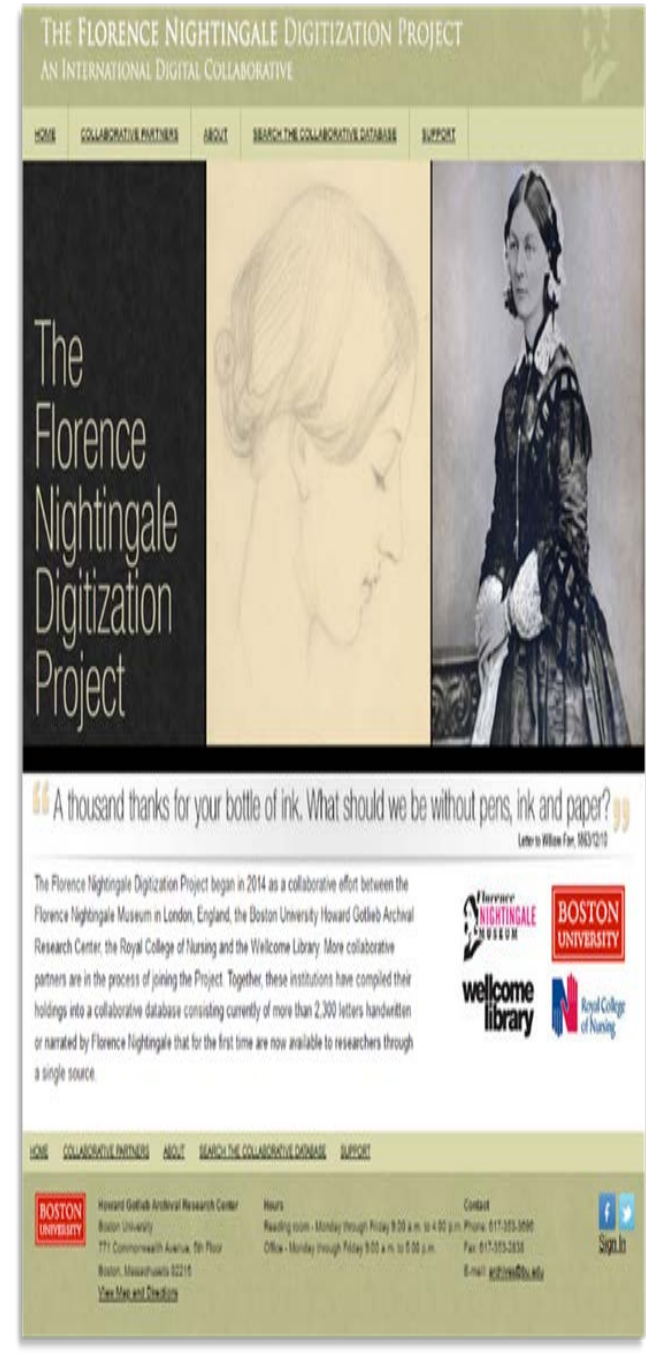

Imagen 5. The Florence Nightingale Project.

Esta Institución posee, además, material gráfico relacionado con el legado de Nightingale, como fotografías y un interesante Álbum recopilado por Sue Goldie (MSS.9106-9109), con material gráfico, recortes de prensa, fotografías y otros materiales ilustrativos de sus actividades asistenciales y sociales, especialmente de la Guerra de Crimea.

Colección de cartas del fondo digitalizado de la wellcome library de londres, 18291910. Temas destacados
- Parthenope Nightingale, hermana de Florence.

- Sir Harry Verney, cuñado de Florence.

- Sir William Aitken, profesor de Patología en la Escuela de Medicina del Ejército (19 cartas).

- George Hanby De'ath, Oficial médico de Salud de Buckingham (19 cartas).

- William Farr (807-1883), estadístico (128 entradas).

- Miss Louisa Gordon, matrona en el Hospital de St Thomas.

- Miss Amy Hughes, Superintendente de la Cooperación de Enfermeras (20 cartas).

- Sir John Henry Lefroy.

- Charles C. Plowden, del Departamento Sanitario de la Oficina de India (30 cartas).

- Mary Clarke Mohl, amiga de Florence.

\section{'Álbum dedicado a Florence Nightingale y su servicio en crimea}

- Recortes de noticias de prensa.

- Cartas de Florence Nightingale a W E Nightingale, Parthenope Nightingale (luego Veney), C H Bracebridge y otros personajes cercanos.

- Dibujos.

- Fotografías.

- Material impreso variado: ilustraciones impresas, un plano del Hospital de Scutari y fotografías de retratos, dibujos y grabados de Florence 
Nightingale (Imagen 6).

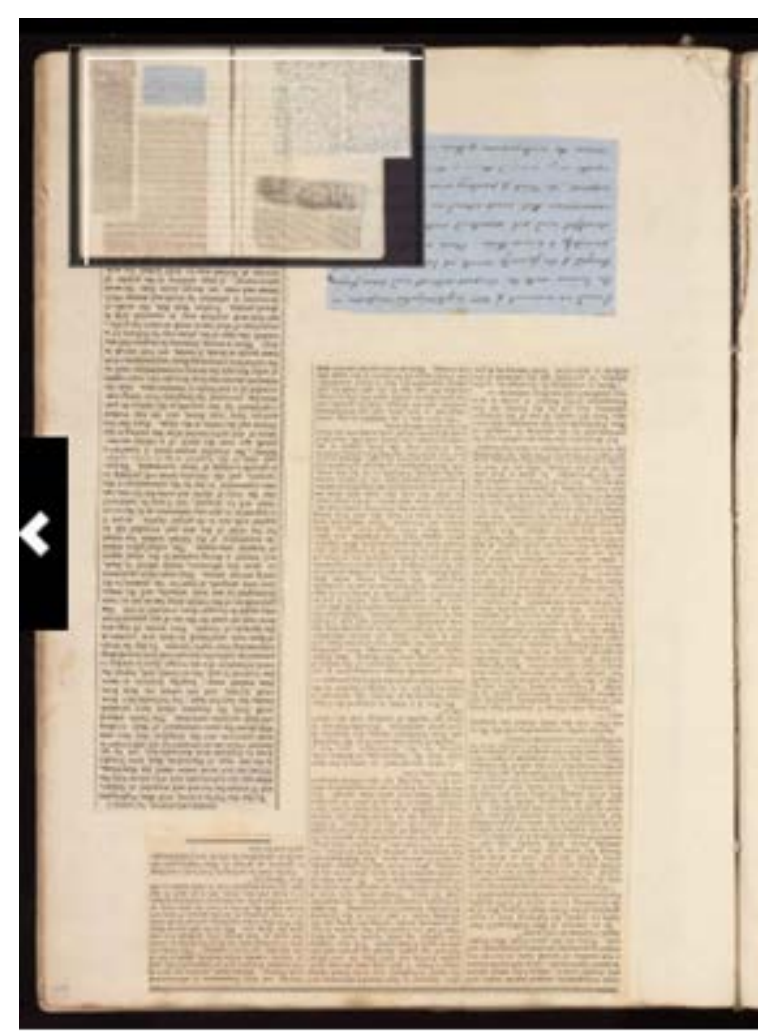

Imagen 6. MS.5484 “Album relating to Florence Nightingale and to her service in the Crimea (18541860)”.

Otro proyecto muy interesante es el que desarrolla la Reynolds-Finley Library, de la Universidad de Alabama, en Birmingham (Estados Unidos).

La Biblioteca Histórica ReynoldsFinley cuenta con una colección de más de 14.000 libros raros, manuscritos, revistas y folletos relacionados con la historia de la medicina, la ciencia y la atención médica, que van cronológicamente desde el siglo XIII hasta mediados del siglo $\mathrm{XX}$, que ofrece un magnífico panorama de aspectos muy diversos sobre temas de Medicina y Salud (Imagen 7).

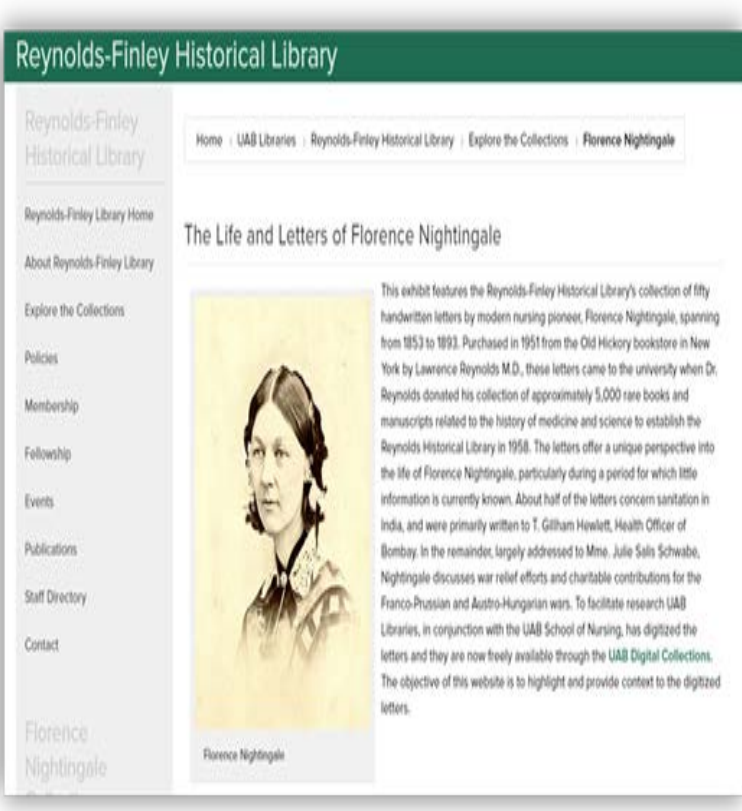

Imagen 7. Sección de la Reynolds-Finley Historical Library dedicada a Florence Nightingale.

Esta colección incluye 50 cartas manuscritas de Florence Nightingale, escritas entre 1853 y 1893, uno de los períodos más interesantes de su vida, por la ingente actividad que desplegó en diversos campos (Imagen 7).

El conjunto de cartas fue adquirido en 1951 en la librería Old Hickory de Nueva York por Lawrence Reynolds MD y llegaron a la Universidad en 1958, cuando el Dr. Reynolds donó su colección personal de aproximadamente 5.000 libros y manuscritos raros relacionados con la historia de la medicina y la ciencia, con la finalidad de establecer la Biblioteca Histórica de Reynolds.

Las cartas ofrecen una perspectiva única de la vida de Florence Nightingale, particularmente durante un período para el cual actualmente se conoce poca información. Alrededor de la mitad de las cartas se refieren al saneamiento en la India, y se enviaron, entre otras personalidades, a Thomas Gillham Hewlett, Oficial de Salud de Bombay.

En el resto, en gran parte dirigido a 

(Universidad de Alicante)

la señora Julie Salis Schwabe, Nightingale analiza los esfuerzos de ayuda de guerra y las contribuciones caritativas para las guerras franco-prusianas y austro-húngaras (Imagen 8).

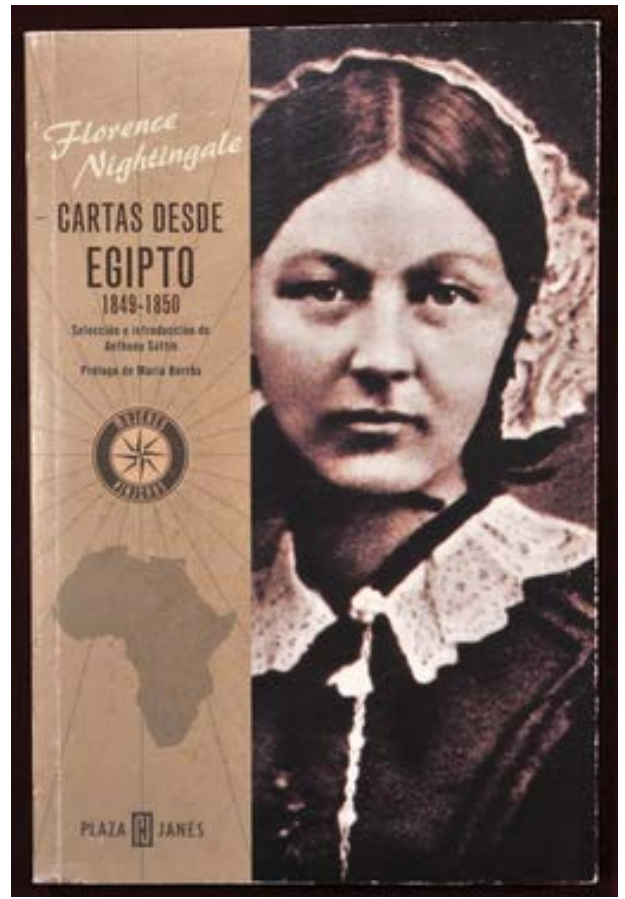

Imagen 8. Portada de la edición de Plaza \& Janés de “Cartas desde Egipto, 1849-1850”, de Florence Nightingale.

Para facilitar la investigación, la Biblioteca de la Universidad de Alabama (UAB), conjuntamente con la Escuela de Enfermería de dicha Universidad, ha digitalizado las cartas y ahora están disponibles en abierto a través de las Colecciones Digitales de la UAB. El objetivo de este sitio web es resaltar y proporcionar un amplio contexto a estas cartas y a su autora.

Para finalizar este apartado, destacamos por su interés en el aspecto humanístico de Florence Nightingale sus Cartas desde Egipto, escritas cuando contaba 29 años, durante un viaje que realizó a este país en compañía de dos amigos, Charles y Selina Bracebridge, entre 1849 y1850. Las cartas van dirigidas especialmente a sus familiares y son habitualmente extensas y descriptivas, refiriendo los paisajes y las costumbres de los egipcios, mostrando a una joven estusiasta de las culturas y pueblos de África y su intensa vida interior (Nightingale, F., 2000a) (Imagen 9).

\section{Florence Nightingale y la prensa: de mujer a heroína y mito universal}

Casi con toda seguridad, no podríamos comprender la figura de Florence Nightingale sin acercarnos a su especial relación con la prensa de la época. Una primera gran muestra de ello fue el seguimiento minucioso que hizo The Times de la participación de la enfermera en la Guerra de Crimea (Goldie, S. M., 1988). Desde el 24 de octubre de 1854, cuando Florence marcha a Escutari con un grupo de enfermeras, por orden de Sir Sidney Herbert, Secretario de Guerra, siendo aún una desconocida para la sociedad Británica, hasta 1856, cuando volvió a casa, fueron dos años que convirtieron a la mujer en heroína. Casi a diario, William Howard Russel, corresponsal de este periódico para cubrir el conflicto bélico, enviaba a Londres sus crónicas sobre todas las actividades que emprendía y desarrollaba Mss. Nightingale, sus preocupaciones por los heridos, por mejorar las enfermerías en donde se recogían los soldados que volvían del frente y sus disputas con los oficiales al mando de las tropas (Coates, T., 2014). La sociedad victoriana leía con auténtica pasión las historias que llegaban desde la primera línea de batalla y terminaron admirando cada día más a la esforzada enfermera. Durante los tres años que duró la contienda, la sociedad británica convirtió a la mujer en “mito”. Una vez de vuelta a casa, Florence Nightingale era una persona reconocida, admirada, respetada y ejemplo de mujer luchadora (Imagen 9). 


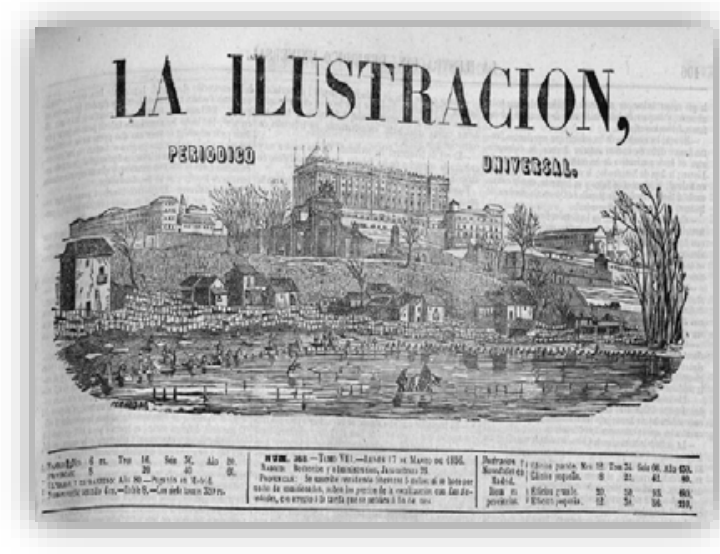

Imagen 9. Portada de "La Ilustración”, publicado el lunes, 17 de marzo de 1856 .

Con la prensa británica volcada completamente con la figura de la enfermera británica, buena parte de la prensa europea, incluyendo la española, copiaba casi literalmente sus crónicas y hacía llegar a sus lectores las historias que se escribían sobre ella (Biblioteca Virtual de la Prensa Histórica, 2021). La prensa española decimonónica recogió numerosas referencias a la vida y a las actividades de Florence Nightingale, que contribuyeron poderosamente a difundir el mito a los lectores españoles. Esto es visible no solamente en la prensa española de ámbito nacional, sino también en la que circuló por un ámbito más local. Publicaciones como La Ilustración. Periódico Universal, que circuló entre 1849 y 1857, se hizo eco de sus actividades y traducía crónicas de The Times para sus lectores. El lunes, 17 de marzo de 1856, La Ilustración publica en su página 110 un extenso artículo titulado "Mis Florence Nightingale”, en donde se resaltan las muchas virtudes que mostraba la enfermera británica, que fueron creando todo un estado de opinión muy favorable hacia su persona entre los lectores españoles.

En otras publicaciones localizamos referencias y noticias sobre Florence Nightingale, como en La Crónica. Diario de avisos, noticias y anuncios, de Huesca.

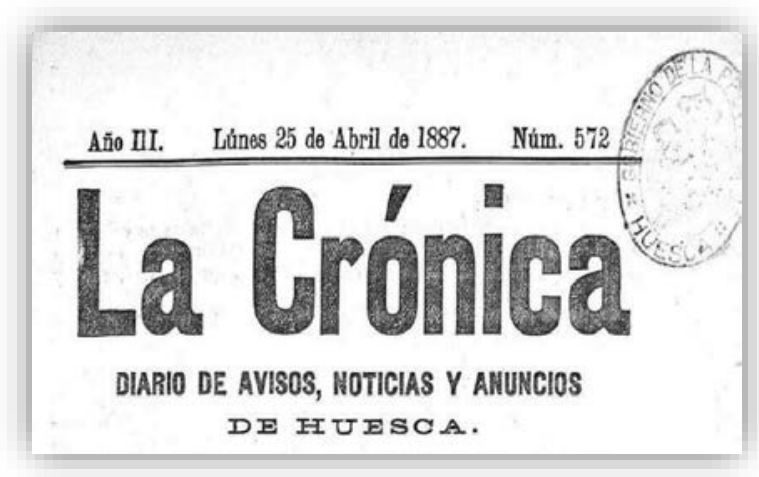

Imagen 10. “La Crónica”, lunes 25 de abril de 1887. Referencias a la enfermera británica

Este diario se hace eco en su número 572, publicado el lunes 25 de abril de 1887, de una Florence Nightingale activista, luchadora y siempre bien relacionada con las grandes personalidades de la alta nobleza británica (Imagen 10). En sus páginas publicó:

"Lo que les falta a las mujeres es el espíritu de iniciativa, la voluntad. En Inglaterra, ha sido menester que señoras de la alta sociedad y un cierto número de hombres de gran posición se pusiesen al frente del movimiento. Entre las fundadoras de las Trade's-unions femeninas citaremos: Madame Paterson, fundadora de la "Liga de protección y de previsión de las mujeres”;

Madame Farvcelt, la esposa del exministro de Correos de Inglaterra; Lady Langton, hermana del duque de Buckingham; las Vizcondesas Amberley y Huberton; Madame Stuart, Mill, viuda del economista; Miss William; Miss Blackburn; Miss. Brown; Miss. Florence Nightingale, tan conocida en la guerra de Crimea, etc. etc. Gracias a tan poderosos concursos, diversos Unions pudieron constituirse. Lord Leicester se puso al frente del movimiento en provincia, donde se constituyeron nueve asociaciones. En Londres hay diez que marchan perfectamente”. 
Similares tratamientos reciben Florence Nightingale en otros diarios o semanarios españoles, como en El Cantábrico. Diario de la mañana, que se imprimía en Santander.

En el siglo XX siguió la prensa española elogiando su vida y su obra, muy especialmente tras su muerte en 1910. El Boletín de la Institución Libre de Enseñanza de 30 de noviembre de 1918, número 704, alude a la influencia de damas británicas en la educación de las jóvenes inglesas y estadounidenses, entre ellas Florence Nightingale.

De unos años después es el amplio artículo que dedica en lengua catalana a la enfermera el semanario Mirador. Setmanari de Literatura, Art i Política, impreso en Barcelona, el jueves 15 de agosto de 1935, número 339, titulado "La Dama de la Llantia. Florence Nightingale, precursora de la Creu Roja”. Un estudio sistemático de la prensa española de la segunda mitad del siglo XIX y todo el siglo XX está mostrando la difusión de la obra de Florence Nightingale en nuestro país y de su importancia en el nacimiento del "mito".

\section{CONCLUSIONES}

La escritura no solamente fue una pasión para Florence Nightingale, sino un poderoso instrumento que supo utilizar para dar forma a su obra enfermera, pero también a su imagen de mujer luchadora $y$ reivindicadora de los derechos de la mujer. Su poderosa imagen fue creciendo a la luz de la prensa británica al servicio del Imperio y ocupó muchas páginas de las lecturas asiduas de las mujeres y hombres de la Inglaterra victoriana, que veía en ella una manera de liberarse de los rígidos convencionalismos de la época a través de su vida. Florence Nightingale fue una mujer que luchó por su libertad de acción y movimiento, nada fácil en la Inglaterra de la segunda mitad del siglo XIX.
La recuperación y difusión del ingente legado escrito a través de los proyectos de digitalización nos está mostrando a una Florence Nightingale que sobrepasa en mucho el marco de la Enfermería por la que ha alcanzado gran parte de su notoriedad mundial y nos acerca a la "mujer" que está más allá de la "enfermera".

Hoy, el legado escrito de Florence Nightingale cobra una nueva dimensión. Con los proyectos de digitalización y su apertura a toda la sociedad, estamos convencidos de que otros muchos aspectos de la poderosa personalidad de la enfermera británica enriquecerán su imagen.

En el contexto de la investigación de la Historia de la Enfermería, la recuperación del legado escrito de Florence Nightingale se ha convertido en todo un referente para que procedamos a la recuperación del legado escrito de otras muchas enfermeras, entre ellas las españolas, y podamos con ello dar una mayor visibilidad a una profesión que lleva siglos dando lo mejor de sí misma a la sociedad a la que presta sus servicios.

\section{BIBLIOGRAFÍA}

Backes, V. M. (2000). El legado histórico del modelo Nightingale: su estilo de pensamiento y praxis. Horizonte de Enfermería, IX (1), 17-21.

Baly, M. E. (1988). Florence Nightingale and the Nursin Legacy. New York: Croom Helm.

Gobierno de España. Ministerio de Cultura y Deporte (2021). Biblioteca Virtual de la Prensa Histórica. Recuperado de https://prensahistorica.mcu.es/es/inicio/inici o.do

Boston University (2020). Disponible en http://archives.bu.edu/web/florencenightingale

Coates, T. (2014). Delane's War: How John 
Delane of The Times Newspaper and Florence Nightingale brought down the British Government. London: Freckle.

Cook, E. (1913). The life of Florence Nightingale. In two volumes. Vol. I (18201861). London: Macmillan and Co., Limited.

Florence Nightingale Museum, Disponible en https://www.florence-nightingale.co.uk/ Goldie, S. M. (1988). I Have Done My Duty: Florence Nightingale in the Crimean War, 1854-56 (The Selected letters of Florence Nightingale). Manchester: Manchester University Press.

Hartill, R. (1996). Florence Nightingale (Visionary Women). Letters and Reflexions. London: Arthur James Ltd.

Jowett, B. (1987). Dear Miss Nightingale: Selection of Letters to Florence Nightingale, 1860-93. Oxford: Clarendon Press.

La Crónica. Diario de avisos, noticias y anuncios. Huesca. Publicado el lunes 25 de abril de 1887, número 572.

La Ilustración. Periodico Universal. Publicado el lunes, 17 de marzo de 1856.

Nightingale, F. (1914). Florence Nightingale to her nurses: a selection from Miss Nightingales's addresses to probationers and nurses of the Nightingale School at St. Thomas's Hospital. London: Macmillan and Co. Limited.

Nightingale, F. (1974). Letters of Florence Nightingale in the history of nursing archive.

Boston: Boston University.

Nightingale, F. (1976). Notes on Hospital. New York: Gordon.

Nightingale, F. (2000a). Cartas desde Egipto(1849-1850). Selección e introducción de Anthony Sattin. Prólogo de María Borras. Barcelona: Plaza y Janés.

Nightingale, F. (2000b). Notas sobre Enfermería. Qué es y qué no es. Barcelona: Masson.

Wake, R. (1998). The Nightingale Training School (1860-1996). London: Haggerston Press. University of Alabama.

Vicinus, M. (1989). Ever Yours, Florence Nightingale. Selected Letters. Ed. By Martya
Vicinus and Bea Nergaard. Marie Marmo

Mullaney, Caldwell Coll., N.J.: Harvard University Press.

Wellcome Library (1). Disponible en https://wellcomelibrary.org/

Wellcome Library (2). Disponible en https://wellcomelibrary.org/

https://wellcomelibrary.org/item/b1929447

$5 \#$ ? $\mathrm{c}=0 \& \mathrm{~m}=0 \& \mathrm{~s}=0 \& \mathrm{cv}=0 \& \mathrm{z}=-$

0.6687\%2C-0.0734\%2C2.3374\%2C1.4683

Woodham-Smith, C. (1992). Florence

Nightingale. Suffolk: Paperback Edition. 